\title{
Introduction: commercialization of knowledge in a regional context: new perspectives
}

\author{
Tüzin Baycan • Roger R. Stough
}

Published online: 27 June 2012

(C) Springer-Verlag 2012

Knowledge is recognized as the crucial element of economic growth in a knowledgebased economy. Scientific knowledge becomes increasingly important for innovation and new business development, and universities play an enhanced role in knowledge production and innovation both as providers of human capital and as seedbeds for new firms. Academic knowledge commercialization is increasingly seen as a potential economic development driver and model, and an associated pattern of transformation toward an entrepreneurial university is observed in many countries. A substantial increase in university support of commercialization and technology transfer is also observed in general.

Despite the increasing importance of knowledge commercialization, the literature provides limited explanation of the relationship between research commercialization and knowledge production and its local and regional diffusion. Although knowledge is understood to be an essential driver of economic growth, the impact of academic entrepreneurial activities including commercialization of academic research is not fully understood.

There are severe limitations and research gaps in the literature on knowledge commercialization. In order to bridge these problems, this Symposium aims to discuss the emerging factors in bridging the gap between knowledge and its commercialization, and to highlight the research agenda and the challenges for the academy, industry and government from a regional development perspective.

\footnotetext{
T. Baycan $(\bowtie)$

Istanbul Technical University, Istanbul, Turkey e-mail: tbaycan@itu.edu.tr; tbaycan@gmu.edu

T. Baycan · R. R. Stough $(\varangle)$

George Mason University, Fairfax, VA, USA

e-mail: rstough@gmu.edu
} 
This Symposium includes a set of earlier papers that were presented in November 2010 in Denver (USA), at a special session on 'Bridging Knowledge to Commercialization: Review and Research Agenda' as part of the 57th Annual North American Meetings of the Regional Science Association International (NARSC).

The collection of papers in this Symposium considers commercialization and valorization of knowledge as an essential element of regional innovation systems and economic development. Therefore, the Symposium addresses modern theories and concepts relating to research on commercialization of knowledge and regional economic development and provides an overview and introduction to this fascinating and rapidly emerging field for academics, policy makers, entrepreneurs, researchers and students who share a common interest and commitment to knowledge commercialization.

The Symposium consists of four papers that highlight the central role of commercialization of knowledge in a regional context from multiple perspectives. In the first paper, Baycan and Stough consider commercialization of knowledge as a complex and multi faceted phenomenon. On the basis of a review of the literature, they investigate the topic from a taxonomic perspective and highlight 'the good', 'the bad' and 'the challenging' in commercialization of knowledge. They first identify and examine concepts that help to bridge the gap from knowledge to commercialization, and then offer a conceptual framework erected on a typology of metaphores for knowledge. Next, they evaluate the societal benefits of knowledge commercialization that they describe as 'the good' and value conflicts and the differences in culture and perspectives that they consider as 'the bad' in commercialization of knowledge. In order to highlight 'the good' and 'the bad', they evaluate the academic, societal, spatial, cultural and ethical implications of commercialization of knowledge from a regional development perspective. Finally, they highlight 'the challenging' for academia, industry and government while describing the critical framework conditions that are needed to effectively foster commercialization of knowledge.

In the second paper, Miller and Acs describe and explain commercialization on the campus. While addressing the past and recent developments in U.S. higher education system, they investigate how the modern 'science of management' has been applied to the university and how a regional and national landscape full of technology transfer programs, triple-helixes, science parks, spin-offs, and corporate and venture partnerships has become the norm in the United States. On the basis of an analysis of past and recent developments in knowledge commercialization, they argue that the campus and its relationship to the broader regional, national and international context has been viewed by institutions, researchers and policy makers from a limited 'management' perspective in attempting to control, direct and understand its economic impact. They underline limitations in the literature and in regional and national policy making and propose an alternative exploratory, organizing framework for the 'commercializing' university in the entrepreneurial age. Against the limitations of the current 'organization-centric commercialization model', they suggest an 'individual-centric commercialization model'.

The next paper by Hemert, Nijkamp and Masurel investigates commercialization through knowledge networks while addressing a recent knowledge circulation 
experiment in the Netherlands that is based on a system of 'innovation vouchers' - a government subsidy programme - to stimulate SMEs to develop innovation in cooperation with knowledge institutes. While using a large sample of Dutch SMEs that have successfully applied for these vouchers, the paper analyzes the innovation strategy of these SMEs on the basis of their sources of innovation, innovation capabilities, innovation performance and commercialization sources. The results show that contacts with universities and international networks positively influence innovation capabilities and innovation performance of Dutch SMEs. The results also show that contacts with competitors which represent one of the major commercialization sources positively influence innovation performance.

The final paper by Goldstein, Bergman and Maier compares EU and US faculty views of university involvement in commercialization and regional economic development. The paper analyzes faculty attitudes towards their universities' involvement in facilitating commercialization of knowledge, and regional economic development, and measures the variation in attitudes among faculty on the basis of individual attributes, institutional characteristics and regional economic conditions. The results of the analysis demonstrate that faculty are significantly more supportive of their universities assisting regional economic development compared to commercialization of knowledge and universities individual and disciplinary factors are more important than institutional and regional economic factors. The results also demonstrate that there are strong similarities between EU and US faculty attitudes.

The overall conclusion of this Symposium is that research in the field of knowledge commercialization does significantly contribute to a better understanding of the complexity of the issues concerned and has contributed to the development of relevant policies for bridging the gap of knowledge to commercialization and regional economic development. The Symposium provides important insights into the ongoing transformation of universities to become more dynamic and entrepreneurial institutions. The Symposium offers many contributions, in particular to academic and social scientists, policy analysts and policy makers, the business sector and society at large and suggests a set of challenges from different regional and structural perspectives. 